ARTICLE

DOI: $10.1038 / \mathrm{s} 41467-018-04246-0$

\title{
Hollow organic capsules assemble into cellular semiconductors
}

Boyuan Zhang ${ }^{1}$, Raúl Hernández Sánchez (10 1,2, Yu Zhong ${ }^{1}$, Melissa Ball', Maxwell W. Terban³, Daniel Paley², Simon J.L. Billinge (i) ${ }^{3,4}$, Fay $\mathrm{Ng}^{1}$, Michael L. Steigerwald ${ }^{1} \&$ Colin Nuckolls (i) ${ }^{1,5}$

Self-assembly of electroactive molecules is a promising route to new types of functional semiconductors. Here we report a capsule-shaped molecule that assembles itself into a cellular semiconducting material. The interior space of the capsule with a volume of $\sim 415 \AA^{3}$ is a nanoenvironment that can accommodate a guest. To self-assemble these capsules into electronic materials, we functionalize the thiophene rings with bromines, which encode selfassembly into two-dimensional layers held together through halogen bonding interactions. In the solid state and in films, these two-dimensional layers assemble into the threedimensional crystalline structure. This hollow material is able to form the active layer in field effect transistor devices. We find that the current of these devices has strong response to the guest's interaction within the hollow spaces in the film. These devices are remarkable in their ability to distinguish, through their electrical response, between small differences in the guest.

\footnotetext{
${ }^{1}$ Department of Chemistry, Columbia University, New York, NY 10027, USA. ${ }^{2}$ Columbia Nano Initiative, Columbia University, New York, NY 10027, USA

${ }^{3}$ Department of Applied Physics and Applied Mathematics, Columbia University, New York, NY 10027, USA. ${ }^{4}$ Condensed Matter Physics and Materials Science Department, Brookhaven National Laboratory, Upton, NY 11973, USA. ${ }^{5}$ The State Key Laboratory of Refractories and Metallurgy, Institute of Advanced Materials and Nanotechnology, School of Chemistry and Chemical Engineering, Wuhan University of Science and Technology, Wuhan 430081, China. These authors contributed equally: Boyuan Zhang, Raúl Hernández Sánchez. Correspondence and requests for materials should be addressed to F.N. (email: fwn2@columbia.edu) or to M.L.S. (email: mls2064@columbia.edu) or to C.N. (email: cn37@columbia.edu)
} 
T here is a growing class of electroactive, conjugated cyclic molecules that are being applied in several areas of materials science ${ }^{1-27}$. These cyclic, conjugated organic semiconductors have interior spaces that should be useful as a locus for guest inclusion to tune the electronic and optoelectronic properties ${ }^{6,22,28-30}$. Conjugated, cyclic semiconductors that incorporate diphenyl perylene diimides (PDIs) have many benefits as the active elements in organic field-effect transistors (OFETs), organic photovoltaics ${ }^{31}$, and organic photodetectors ${ }^{32}$. In the cases we have studied until now, the diphenyl-PDI subunits provide open macrocycles with cavity diameters so large that they exhibit dynamic stereochemistry as the PDI subunits readily rotate through the interior of the ring ${ }^{20}$.

We describe here a new electronic material whose molecular components are shape persistent and can be functionalized so they self-assemble into semiconducting films. These films feature regular open spaces, cells, in them. We call this new material a cellular organic semiconductor. The molecular substructure results from the union of bithiophenes (B) and PDI into a macrocycle. We fully utilize the interior of the macrocycle in devices, and endow the materials with permanent, open voids, by rigidifying the macrocycle so they cannot collapse. We describe the syntheses and structures of the organic semiconductors shown in Fig. 1a, b: the trimeric macrocycle 1, (-PDI-B-) $)_{3}$ and its brominated version $1-\mathrm{Br}_{12}$. These macrocycles exist as a single pair of enantiomers and are shape persistent to temperatures above $160^{\circ} \mathrm{C}$. Their capsular structure is capped on the ends by the alkyl sidechains and on the equator by the electronic components (the PDI and B subunits). This is shown in Fig. 1c-h. While $\mathbf{1}$ and $\mathbf{1}-\mathrm{Br}_{12}$ share the same overall shape and molecular structure, compound $\mathbf{1}$ does not organize well in films or in the solid state. However, the twelve bromines of $\mathbf{1}-\mathrm{Br}_{12}$ participate in halogen bonding interactions, facilitating self-assembly in films and crystals. 1- $\mathrm{Br}_{12}$ 's self-organization creates films that have regular voids in them, forming the hollow organic semiconducting phase. The remarkable finding is that $1-\mathrm{Br}_{12}$ 's cellular films act as the active layer in OFETs, and the electrical response depends on the guest that occupies the interior space.

\section{Results}

Capsule construction. We developed a synthesis of 1 based on our own previous studies to make the diphenyl-PDI macrocycles ${ }^{20}$ that builds from the methodology originally pioneered for cyclothiophenes and later for cycloparaphenylenes ${ }^{20,31}$. The Supporting Information contains the details of the syntheses and characterization (Supplementary Methods and Supplementary Fig. 1-3) of $\mathbf{1}$ and $\mathbf{1}-\mathrm{Br}_{12}$. For each of the macrocycles, the 1,7-dithienyl-PDI subunit introduces an element of chirality because it can exist in either a $R$ - or $S$-helical conformation. This allows for the possibility of two pairs of enantiomers (RRR/SSS and RRS/SSR $)^{20}$. However, in the reaction to form 1 , we only
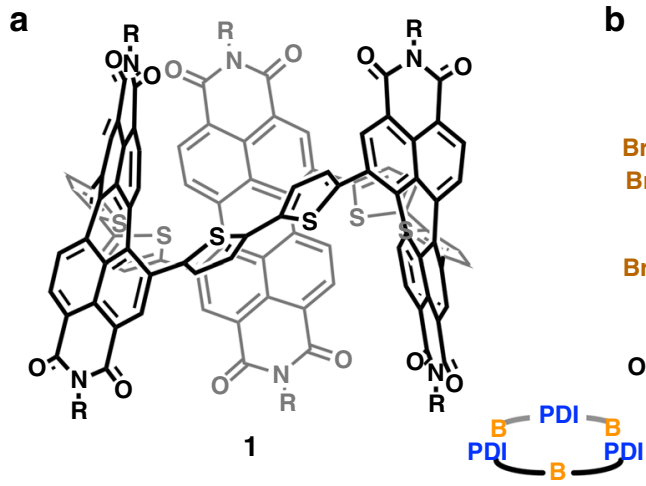

b

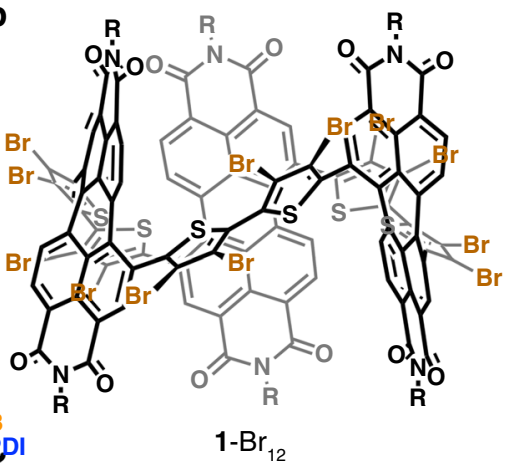

$\mathrm{R}=\mathrm{CH}\left(\mathrm{C}_{5} \mathrm{H}_{11}\right)_{2}$
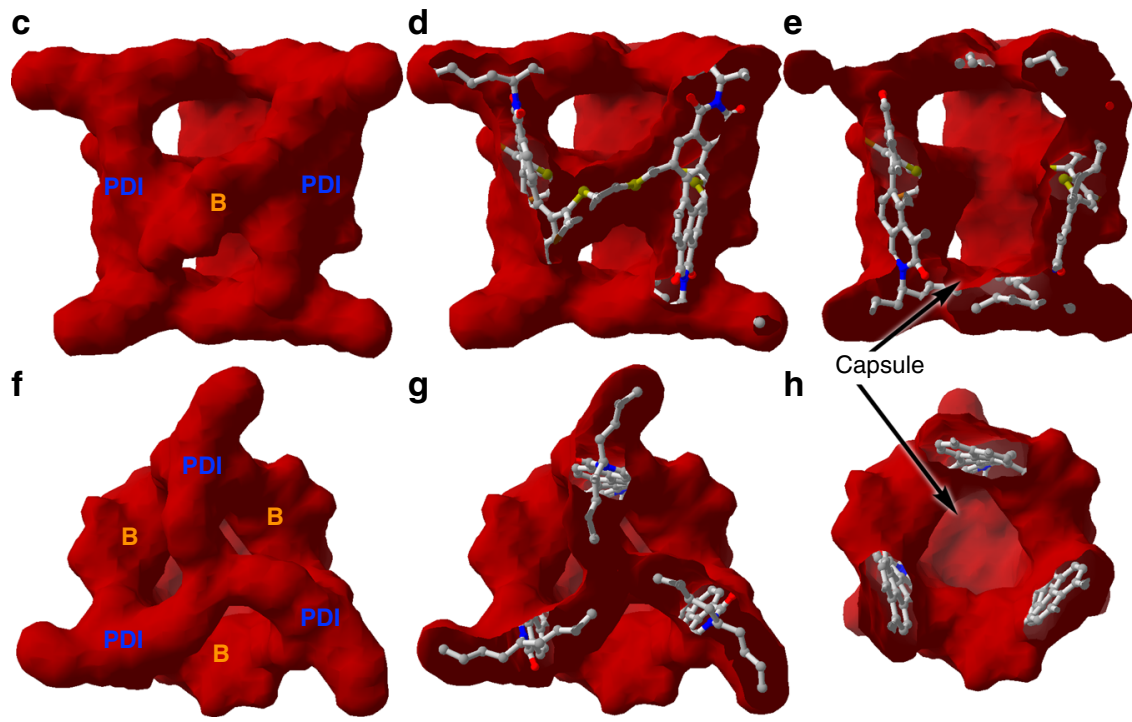

Fig. 1 Structures of cellular semiconductors. a Trimer $\mathbf{1}$; and $\mathbf{b} \mathbf{1}-\mathrm{Br}_{12}$. Van der Waals Surface of $\mathbf{1}-\mathrm{Br}_{12}$ seen from the side (c), and top (f). In the sequence $\mathbf{c}-$ $\mathbf{e}$ and $\mathbf{f}-\mathbf{h}$ the molecule is trimmed down to expose its cavity or capsule 

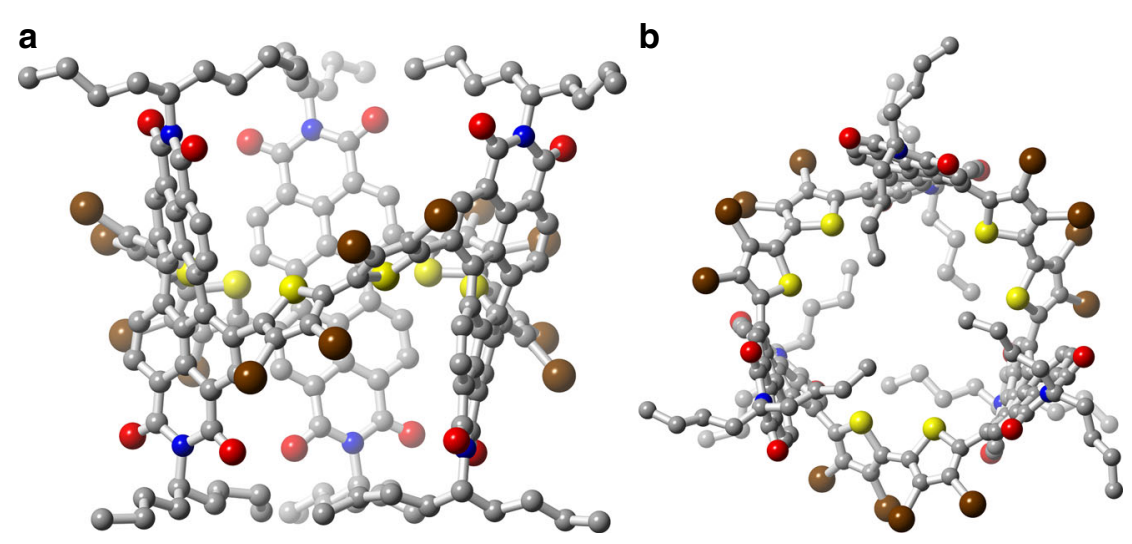

Fig. 2 Molecular structure from SCXRD of $\mathbf{1}-\mathrm{Br}_{12}$. a Side and $\mathbf{b}$ top view of (SSS)-1- $\mathrm{Br}_{12} . \mathrm{C}, \mathrm{N}, \mathrm{O}, \mathrm{S}$, and $\mathrm{Br}$ atoms are colored in gray, blue, red, yellow, and brown, respectively. Hydrogen atoms have been removed to clarify the view. The alkyl chains on the imide are refined to only nine of the eleven carbon atoms due to disorder (see Supplementary Methods)

observe the RRR/SSS pair. We introduce bromine atoms in the thiophene rings of $\mathbf{1}$ to encourage self-assembly through halogen bonding interactions ${ }^{33}$.

To test the shape persistence of these macrocycles, we separate the two enantiomers of $\mathbf{1}$, using a chiral stationary phase for HPLC, and monitor their interconversion as we heat the samples. The two enantiomers of $\mathbf{1}$ exhibit an intense (and opposite) chiroptic response in their circular dichroism spectra (Supplementary Fig. 2). Remarkably, the enantiomers of $\mathbf{1}$ do not interconvert, even when heated up to $160^{\circ} \mathrm{C}$. Macrocycle $\mathbf{1}$ is the first PDI-based cyclic semiconductor that can be isolated in its optically active form and is shape-persistent.

Capsule structure. The crystals of $\mathbf{1}$, while sizable and faceted, do not diffract well enough to yield a structure from single-crystal Xray diffraction (SCXRD), but we were able to grow single crystals of sufficient quality to yield the structures of $1-\mathrm{Br}_{12}$. Figure $2 \mathrm{a}, \mathrm{b}$ displays the structure of one of the two enantiomers of $1-\mathrm{Br}_{12}$ $\left[(S S S)-\mathbf{1}-\mathrm{Br}_{12}\right]$. Both enantiomers of $\mathbf{1}-\mathrm{Br}_{12}$ are present in the crystalline state. The structure of $\mathbf{1}-\mathrm{Br}_{12}$ is cylindrical with the three sets of bithiophenes and three PDIs forming the walls at the equator. The ends of the cylinder are capped with branched alkyl chains (Fig. 2 and highlighted in green in Fig. 3a). This creates windows on the side of the structure, displayed in Figs. 1c, $\mathrm{f}$ and 2a. We estimate the interior volume of the capsule in $\mathbf{1 - B r}$ (shown in Fig. 1e, h) to be approximately $415 \AA^{334}$.

Cellular solids from capsular nanostructures. The packing structure in the solid state of $1-\mathrm{Br}_{12}$ (Fig. 3) reveals why the bromines were necessary for long range crystallinity. The structure is composed of sheets of a honeycomb-like arrangement in the ab plane (Fig. 3a). The interactions that bind the cylinders within the $\mathrm{ab}$ plane are from neighboring brominated thiophene rings ( $4.7 \AA$ apart, marked in red in Fig. $3 a, c-d)$ and some secondary $\pi-\pi$ contacts between those same rings (marked in blue in Supplementary Fig. 4). The PDIs do not $\pi$-stack with each other. Instead halogen bonding from the functionalized thiophenes drives the self-assembly process. The cavity of $1-\mathrm{Br}_{12}$ (labeled "Capsule" in Fig. 3a, b) is $\sim 11.4 \AA$ in diameter and is a three-fold symmetric chiral nanoenvironment for guest incorporation within the two-dimensional layer. Due to the packing of the subunits of $1-\mathrm{Br}_{12}$ into a hexameric cyclic structure, a second cavity forms at the center of each hexagon (labeled $i$ in Fig. 3a, b). This cavity is not elongated compared to the capsule's cavity, and each one accounts for $\sim 110 \AA^{3}$ (Supplementary Fig. 5). These honeycomb two-dimensional, cellular sheets then stack through the packing of the alkyl side chains of the imides, (shown in green in Fig. 3). This packing arrangement propagates along the $c$ axis, hinting that these materials could likely be exfoliated to yield molecularly thin sheets of $\mathbf{1}-\mathrm{Br}_{12}$.

We find that $1-\mathrm{Br}_{12}$ self-assembly in cast thin films and powder samples is analogous to what we described above for the single crystal. Supplementary Figs 6, 7 compare the thin film and powder diffraction data for $\mathbf{1}-\mathrm{Br}_{12}$ with the simulated pattern calculated from the SCXRD data. No other reflections are present in the films or powders indicating that the self-assembly motif using the halogen bonding is robust. In order to extract quantitative information about the long-range crystallinity of these new hollow semiconductors we performed pair distribution function (PDF) analyses on powders of $\mathbf{1}$ and 1- $\mathrm{Br}_{12}$ (Supplementary Figs. 8-12). Supplementary Fig. 12 displays the PDF of $1-B_{12}$ at low $\left(1.81 \AA^{-1}\right)$ and high $\left(6.33 \AA^{-1}\right)$ $Q_{\max }$ to give a visualization of the coherence length. The lower and upper bounds of the coherence length of $1-\mathrm{Br}_{12}$ are 335 and $385 \AA$, respectively (Supplementary Fig. 12). To put these values in perspective, this indicates that crystalline domains within 1$\mathrm{Br}_{12}$ contain on average up to 23 to 27 capsules arranged in one direction. In contrast, $\mathbf{1}$, with poor crystallinity and an inability to self-organize (Supplementary Fig. 6), is essentially amorphous. The analysis shows coherence lengths ranging from 65 to $120 \AA$ (Supplementary Fig. 11), which is only a few capsules in length.

Electron transport through cellular films. Figure 4a displays transfer curves from an OFET constructed using a self-assembled thin film of $1-\mathrm{Br}_{12}$. Details for the device dimensions and its properties can be found in the Supplementary Methods. The device exhibits electron transporting character and has a mobility of $\sim 1.5 \times 10^{-2} \mathrm{~cm}^{2} \mathrm{~V}^{-1} \mathrm{~s}^{-1}$. The mobility of $1-\mathrm{Br}_{12}$ is more than 20 times greater than that of $\mathbf{1}\left(\sim 6.8 \times 10^{-4} \mathrm{~cm}^{2} \mathrm{~V}^{-1} \mathrm{~s}^{-1}\right)$ (Supplementary Fig. 13). We attribute this to the robust self-assembly process for $1-\mathrm{Br}_{12}$. From atomic force microscope height images, films of 1 display a smooth surface with root mean square (RMS) roughness of $0.347 \mathrm{~nm}$; in contrast $\mathbf{1}-\mathrm{Br}_{12}$ displays a larger RMS roughness of $3.2 \mathrm{~nm}$, presumably due to its more crystalline nature and better self-assembly properties (Supplementary Fig. 14).

The exciting finding is that the devices show modulation in the drain current depending on what, if anything, is within the voids in the cellular film. Details of the experimental setup to measure the exposure to gases are given in the Supplementary Methods. Every potential guest tested had a measurable effect on the drain current in the device, but the absolute levels of the drain current 

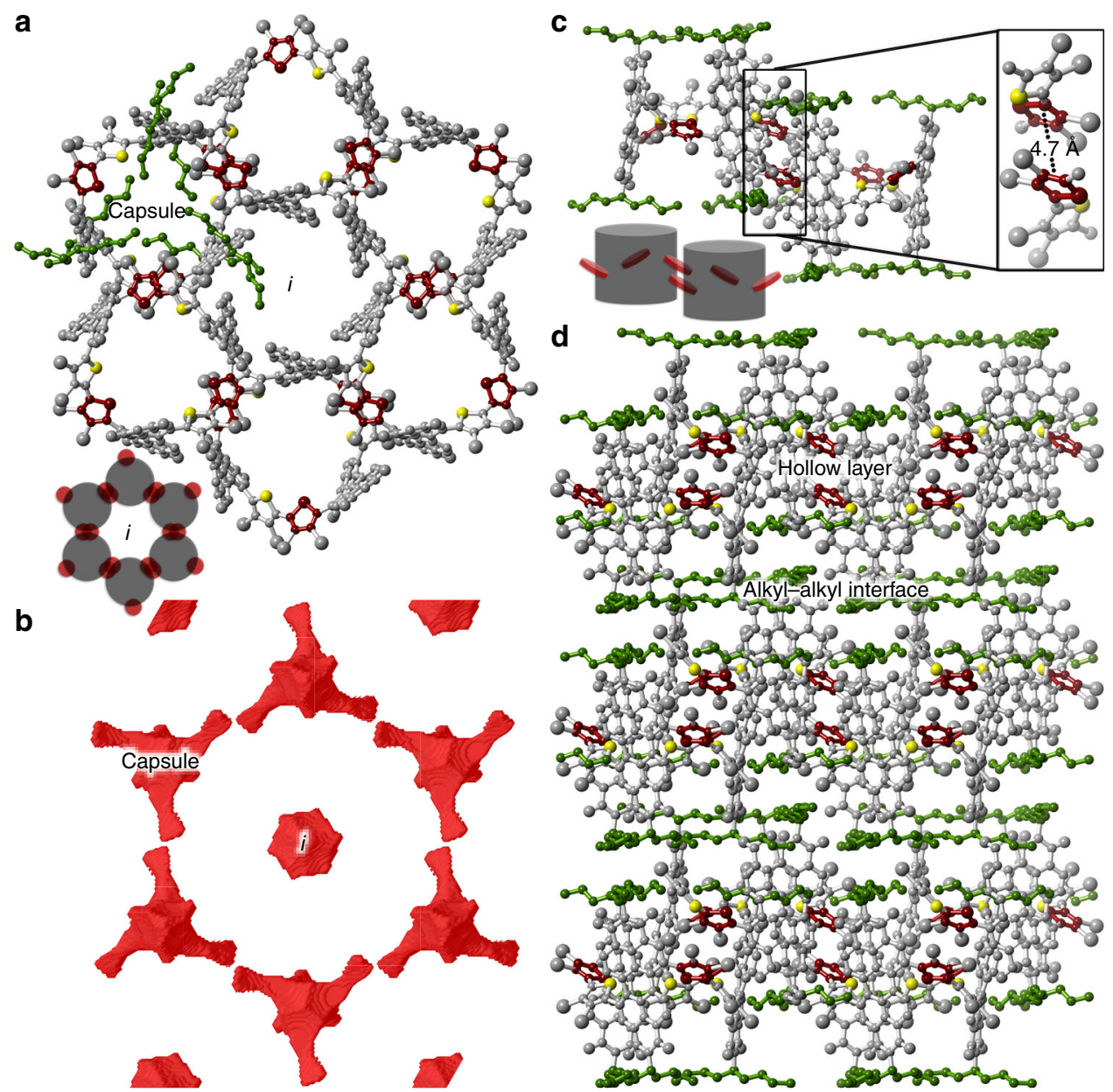

Fig. 3 Structural packing of $\mathbf{1}-\mathrm{Br}_{12}$. a View of the honeycomb structure in the ab plane for $\mathbf{1}-\mathrm{Br}_{12}$. The capsule and $i$ corresponds to the internal cavity of $\mathbf{1}$ $\mathrm{Br}_{12}$ and the cavity formed by the packing of $\mathbf{1}-\mathrm{Br}_{12}$, respectively. The remaining sulfur atoms are colored in yellow to provide a marker to identify the macrocycle cavities. See bottom left cartoon. Highlighted in green are the imide side chains (some of the sidechains have been removed to clarify the view

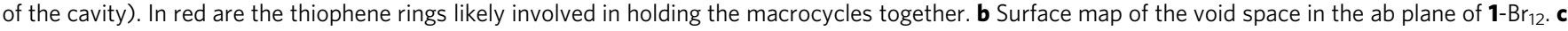
Two molecules of $\mathbf{1}-\mathrm{Br}_{12}$ where the thiophene-to-thiophene interaction is highlighted as an inset. Bottom left cartoon represents this interaction. $\mathbf{d}$ View of the packing of $\mathbf{1}-\mathrm{Br}_{12}$. As shown, the vertical stacking follows the $c$ axis. The alkyl sidechains of the imide are shown in green. Hydrogen atoms have been removed from all structures to clarify the view
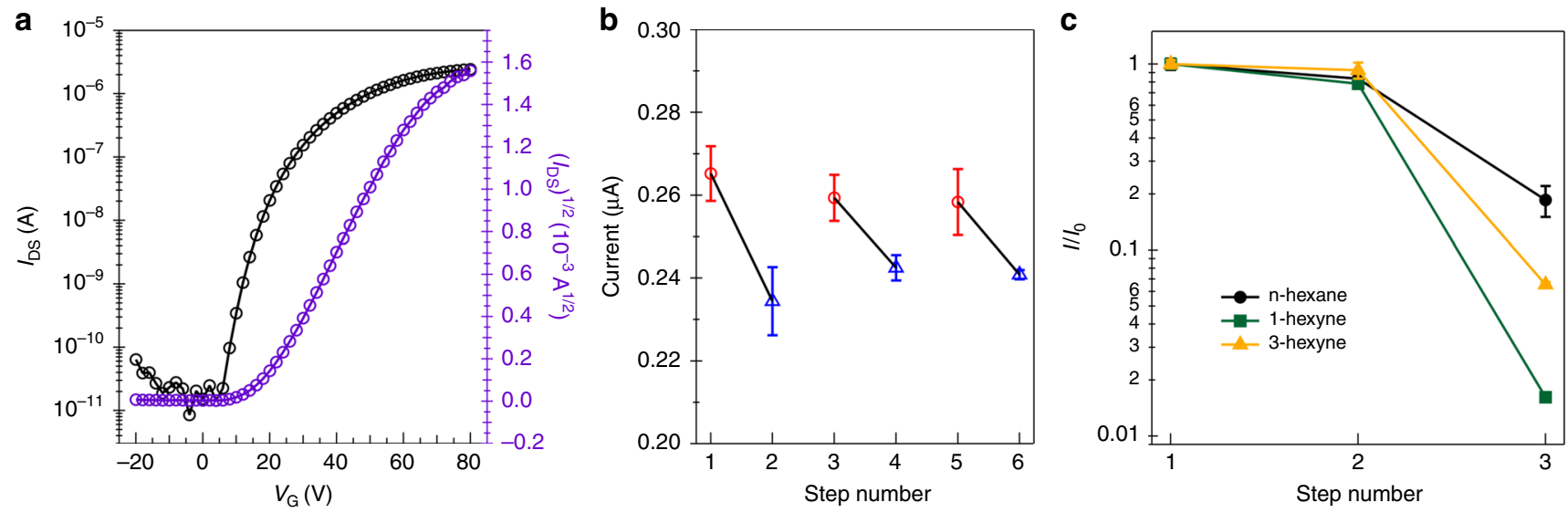

Fig. 4 Electron transport for cellular films. a Transfer characteristics of OFET device for 1- $\mathrm{Br}_{12}$. b Device cycling response under vacuum (red circles) and $\mathrm{N}_{2}$ atmosphere (blue triangles). c Normalized behavior of the device response under vacuum (step 1), $N_{2}$ (step 2), and different analytes atmosphere (step 3 : n-hexane, 3-hexyne and 1-hexyne). Error bars represent the standard error obtained in three measurements 
varied depending on the guest. Figure $4 \mathrm{~b}$ is a representative data set for a device measured sequentially in steps as the atmosphere is changed between vacuum and $\mathrm{N}_{2}$. In certain cases, traditional OFETs show a differential response to nitrogen and vacuum that arises from extrinsic effects (moisture, oxygen, or dielectric effects) ${ }^{35,36}$. We can test the importance of the cellular semiconductor's response by making a control FET device from 1, which does not self-organize into cellular semiconducting films or crystals. We observe no response when comparing its response to nitrogen and vacuum (Supplementary Fig. 15a).

Incorporating more polarizable and functional guests causes more pronounced changes in the drain current. Supplementary Fig. 15b compares the effects of several different guests with a variety of functional groups such as ketones, alcohols, nitriles, alkynes, and alkanes. In each case we are able to differentiate the guest by the current in the device. We highlight here one striking example of how this material responds to a series of closely related hydrocarbons. These hydrocarbons were chosen so that their length, size, and polarity were roughly similar to that of $\mathrm{n}$-hexane, while having an additional functional group in them. Fig. $4 \mathrm{c}$ compares the transistor output for three devices exposed to n-hexane, 3-hexyne, or 1-hexyne, carried by nitrogen under their saturated vapor pressure $\left(148,99\right.$, and $138 \mathrm{mmHg}$ at $25^{\circ} \mathrm{C}$, respectively $)^{37}$. Remarkably, the devices can easily detect these three materials and distinguish them from one another. There is also specificity towards particular analytes; the trend in the device responses does not simply follow the vapor pressure. For $\mathrm{n}$-hexane and 3-hexyne the data was collected after the OFET was in contact with the vapor for $\sim 70 \mathrm{~min}$ (Supplementary Fig. 16a). After this time, the drain current no longer decreased. The original current levels for n-hexane and 3-hexyne could be restored by placing the devices in vacuum (Supplementary Fig. 16b). During these long exposures, we speculate that the guests infiltrate the films to reside in the active part of the films at the gate dielectric interface ${ }^{38,39}$.

We tested the sensitivity of these hollow films and find that there is a linear response between vacuum and 20 part per thousand of the analyte in the atmosphere, after we observed a plateau region as the concentration reached saturation (Supplementary Fig. 17). Access to the porous network formed by the capsules interior $\left(\sim 415 \AA^{3}\right)$ and the $i$-sites $\left(\sim 110 \AA^{3}\right)$ is granted by the windows in the cellular structure as shown in Fig. 1c-h. Using BET, we find that powders of $1-\mathrm{Br}_{12}$ have a surface area of $20 \mathrm{~m}^{2}$ $\mathrm{g}^{-1}$ (versus $1.2 \mathrm{~m}^{2} \mathrm{~g}^{-1}$ for $\mathbf{1}$ ). As $\mathrm{n}$-hexane has a van der Waals molecular volume of $\sim 113 \AA^{340}$, the implication is that $n$-hexane would not fit into the $i$-site. In addition, most of the cellular nature of self-assembled $\mathbf{1}-\mathrm{Br}_{12}$ comes from the capsule interior which is present in a 2:1 numerical ratio relative to the $i$-site. Given the 8-fold difference in volume between these two cavities, we speculate that guests can be accommodated most feasibly at the capsules' interior.

1-hexyne behaves differently than each of the other guests tested. For the devices in an atmosphere of 1-hexyne, the current continues to drop and does not reach a plateau even at times that exceed $2 \mathrm{~h}$ of exposure. In addition, the 1-hexyne devices do not recover to their original levels when placed in vacuum (Supplementary Fig. 18). We speculate that the terminal alkyne is undergoing a reaction under the device conditions that is not possible with the internal alkyne or the alkane. This offers the intriguing possibility that in addition to sensing, the nanoenvironments in these hollow semiconductors can be used as nanoreactors.

\section{Discussion}

We have described here a shape persistent, hollow macrocycle that self-assembles both in the solid state and thin films to form cellular organic semiconductors. The macrocycle is chiral and conformationally locked into a capsular structure, with $\sim 415 \AA^{3}$ volume within its interior. The self-assembly of a brominated derivative of the trimer into cellular films forms the active layer in an organic field-effect transistor device. Once assembled, these films have periodic, nanoscopic, cellular voids. Because the macrocyclic component in the film is conformationally locked, the self-assembled films maintain their interior, open spaces. The hollow films of $1-\mathrm{Br}_{12}$ are responsive to the atmosphere in which the OFETs are measured. These studies chart a clear path to using the interior of the cellular organic semiconductors as gas sensors $^{41,42}$ and nanoreactors.

Data availability. All data are available from the authors upon reasonable request. The X-ray crystallographic coordinates for structures reported in this study have been deposited at the Cambridge Crystallographic Data Centre (CCDC), under deposition number 1834626 . These data can be obtained free of charge from The Cambridge Crystallographic Data Centre via www.ccdc.cam.ac.uk/data_request/cif.

Received: 2 October 2017 Accepted: 10 April 2018

Published online: 16 May 2018

\section{References}

1. Krömer, J. et al. Synthesis of the first fully a-conjugated macrocyclic oligothiophenes: cyclo[n]thiophenes with tunable cavities in the nanometer regime. Angew. Chem. Int. Ed. 39, 3481-3486 (2000).

2. Nakao, K. et al. Giant macrocycles composed of thiophene, acetylene, and ethylene building blocks. J. Am. Chem. Soc. 128, 16740-16747 (2006).

3. Jasti, R., Bhattacharjee, J., Neaton, J. B. \& Bertozzi, C. R. Synthesis, characterization, and theory of [9]-, [12]-, and [18]cycloparaphenylene: carbon nanohoop structures. J. Am. Chem. Soc. 130, 17646-17647 (2008).

4. Zhang, F., Götz, G., Winkler, H. D. F., Schalley, C. A. \& Bäuerle, P. Giant cyclo [n]thiophenes with extended $\pi$ conjugation. Angew. Chem., Int. Ed. 48, 6632-6635 (2009).

5. Omachi, H., Matsuura, S., Segawa, Y. \& Itami, K. A modular and size-selective synthesis of [n] cycloparaphenylenes: a step toward the selective synthesis of [n, n] single-walled carbon nanotubes. Angew. Chem. Int. Ed. 49, 10202-10205 (2010).

6. Iwamoto, T., Watanabe, Y., Sadahiro, T., Haino, T. \& Yamago, S. Size-selective encapsulation of C60 by [10]cycloparaphenylene: formation of the shortest fullerene-peapod. Angew. Chem., Int. Ed. 50, 8342-8344 (2011).

7. Iwamoto, T., Watanabe, Y., Sakamoto, Y., Suzuki, T. \& Yamago, S. Selective and random syntheses of $[n]$ cycloparaphenylenes $(n=8-13)$ and size dependence of their electronic properties. J. Am. Chem. Soc. 133, 8354-8361 (2011).

8. Segawa, Y. et al. Concise synthesis and crystal structure of [12] cycloparaphenylene. Angew. Chem. Int. Ed. 50, 3244-3248 (2011).

9. Yasutomo, S., Petr, Š., Sanae, M., Haruka, O. \& Kenichiro, I. [9] Cycloparaphenylene: nickel-mediated synthesis and crystal structure. Chem. Lett. 40, 423-425 (2011).

10. Sprafke, J. K. et al. Belt-shaped $\pi$-systems: relating geometry to electronic structure in a six-porphyrin nanoring. J. Am. Chem. Soc. 133, 17262-17273 (2011).

11. Hitosugi, S., Nakanishi, W. \& Isobe, H. Atropisomerism in a belt-persistent nanohoop molecule: rotational restriction forced by macrocyclic ring strain. Chem. Asian J. 7, 1550-1552 (2012).

12. Hitosugi, S., Yamasaki, T. \& Isobe, H. Bottom-up synthesis and thread-inbead structures of finite $(\mathrm{n}, 0)$-zigzag single-wall carbon nanotubes. J. Am. Chem. Soc. 134, 12442-12445 (2012).

13. Ishii, Y. et al. Size-selective synthesis of [9]-[11] and [13]cycloparaphenylenes. Chem. Sci. 3, 2340-2345 (2012).

14. Kayahara, E., Sakamoto, Y., Suzuki, T. \& Yamago, S. Selective synthesis and crystal structure of [10]cycloparaphenylene. Org. Lett. 14, 3284-3287 (2012)

15. Omachi, H., Segawa, Y. \& Itami, K. Synthesis of cycloparaphenylenes and related carbon nanorings: a step toward the controlled synthesis of carbon nanotubes. Acc. Chem. Res. 45, 1378-1389 (2012).

16. Evans, P. J., Darzi, E. R. \& Jasti, R. Efficient room-temperature synthesis of a highly strained carbon nanohoop fragment of buckminsterfullerene. Nat. Chem. 6, 404-408 (2014) 
17. Kayahara, E., Patel, V. K. \& Yamago, S. Synthesis and characterization of [5]cycloparaphenylene. J. Am. Chem. Soc. 136, 2284-2287 (2014).

18. Yamago, S., Kayahara, E. \& Iwamoto, T. Organoplatinum-mediated synthesis of cyclic $\pi$-conjugated molecules: towards a new era of three-dimensional aromatic compounds. Chem. Rec. 14, 84-100 (2014).

19. Asai, K., Fukazawa, A. \& Yamaguchi, S. A cyclic octithiophene containing [small beta],[small beta] [prime or minute]-linkages. Chem. Commun. (Camb., U. K.) 51, 6096-6099 (2015).

20. Ball, M. et al. Chiral conjugated corrals. J. Am. Chem. Soc. 137, 9982-9987 (2015).

21. Chang, S.-W. \& Horie, M. A donor-acceptor conjugated block copolymer of poly(arylenevinylene)s by ring-opening metathesis polymerization. Chem. Commun. (Camb., U. K.) 51, 9113-9116 (2015).

22. Chen, Q. et al. Strain-induced stereoselective formation of blue-emitting cyclostilbenes. J. Am. Chem. Soc. 137, 12282-12288 (2015).

23. Darzi, E. R. et al. Synthesis, properties, and design principles of donor-acceptor nanohoops. ACS Cent. Sci. 1, 335-342 (2015).

24. Ito, H., Mitamura, Y., Segawa, Y. \& Itami, K. Thiophene-based, radial $\pi$-conjugation: synthesis, structure, and photophysical properties of cyclo-1,4-phenylene-2', $5^{\prime}$-thienylenes. Angew. Chem. Int. Ed. 54, 159-163 (2015).

25. Jiang, H.-W. et al. Cyclic 2,12-porphyrinylene nanorings as a porphyrin analogue of cycloparaphenylenes. J. Am. Chem. Soc. 137, 2219-2222 (2015).

26. Kuwabara, T., Orii, J., Segawa, Y. \& Itami, K. Curved oligophenylenes as donors in shape-persistent donor-acceptor macrocycles with solvatofluorochromic properties. Angew. Chem. Int. Ed. 54, 9646-9649 (2015).

27. Van Raden, J. M., Darzi, E. R., Zakharov, L. N. \& Jasti, R. Synthesis and characterization of a highly strained donor-acceptor nanohoop. Org. Biomol. Chem. 14, 5721-5727 (2016).

28. Iyoda, M., Yamakawa, J. \& Rahman, M. J. Conjugated macrocycles: concepts and applications. Angew. Chem. Int. Ed. 50, 10522-10553 (2011).

29. Iwamoto, T. et al. Size- and orientation-selective encapsulation of C70 by cycloparaphenylenes. Chem. Eur. J. 19, 14061-14068 (2013).

30. Nakanishi, Y. et al. Size-selective complexation and extraction of endohedral metallofullerenes with cycloparaphenylene. Angew. Chem., Int. Ed. 53, 3102-3106 (2014).

31. Ball, M. et al. Macrocyclization in the design of organic n-type electronic materials. J. Am. Chem. Soc. 138, 12861-12867 (2016).

32. Zhang, B. et al. Rigid, conjugated macrocycles for high performance organic photodetectors. J. Am. Chem. Soc. 138, 16426-16431 (2016).

33. Metrangolo, P., Resnati, G., Pilati, T. \& Biella, S. In Halogen Bonding: Fundamentals and Applications (eds Metrangolo, P. \& Resnati, G.) 105-136 (Springer, Berlin, Heidelberg, 2008).

34. Dolomanov, O. V., Bourhis, L. J., Gildea, R. J., Howard, J. A. K. \& Puschmann, H. OLEX2: a complete structure solution, refinement and analysis program. J. Appl. Crystallogr. 42, 339-341 (2009).

35. Pannemann, C., Diekmann, T. \& Hilleringmann, U. On the degradation of organic field-effect transistors. Proc. 16th International Conference on Microelectronics (ed. Masmoudi, M.) 76-79 (Nouha Editions, Tunis, 2004).

36. Wei-Cheng, S. et al. Characterization of intrinsic hysteresis of pentacenebased organic thin-film transistor through in-situ real-time electrical measurement. Jpn. J. Appl. Phys. 53, $03 \mathrm{CC03}$ (2014).

37. Yaws, C. L. \& Satyro, M. A. In The Yaws Handbook of Vapor Pressure 2nd edn (ed. Yaws, C. L.) Ch. 1 (Gulf Professional Publishing, Houston, 2015).

38. Hsieh, J. C., Liu, C. J. \& Ju, Y. H. Response characteristics of lead phthalocyanine gas sensor: effects of film thickness and crystal morphology. Thin Solid Films. 322, 98-103 (1998).

39. Sakai, G., Matsunaga, N., Shimanoe, K. \& Yamazoe, N. Theory of gasdiffusion controlled sensitivity for thin film semiconductor gas sensor. Sens. Actuators B 80, 125-131 (2001).

40. Zhao, Y. H., Abraham, M. H. \& Zissimos, A. M. Fast calculation of van der waals volume as a sum of atomic and bond contributions and its application to drug compounds. J. Org. Chem. 68, 7368-7373 (2003).
41. Crone, B. et al. Electronic sensing of vapors with organic transistors. Appl. Phys. Lett. 78, 2229-2231 (2001).

42. Huang, W. et al. Highly sensitive NH3 detection based on organic field-effect transistors with tris(pentafluorophenyl)borane as receptor. J. Am. Chem. Soc. 134, 14650-14653 (2012).

\section{Acknowledgements}

Primary support for this project was provided by the Chemical Sciences, Geosciences and Biosciences Division, Office of Basic Energy Sciences, U.S. Department of Energy (DOE), under award number DE-FG02-01ER15264. PDF measurement and analysis (M.W.T. and S.J.L.B.) was supported by the NSF MRSEC program through Columbia in the Center for Precision Assembly of Superstratic and Superatomic Solids (DMR-1420634). The Columbia University Shared Materials Characterization Laboratory (SMCL) was used extensively for this research. We are grateful to Columbia University for the support of this facility. C.N. thanks Sheldon and Dorothea Buckler for their generous support. R.H.S. acknowledges the support from the Columbia Nano Initiative Postdoctoral Fellowship. This research used beamline 28-ID-2 of the National Synchrotron Light Source II, a U.S. Department of Energy (DOE) Office of Science User Facility operated for the DOE Office of Science by Brookhaven National Laboratory under Contract No. DESC0012704.

\section{Author contributions}

F.N., M.L.S. and C.N. conceived the idea. B.Z. and R.H.S. designed the experiments. B.Z. performed device fabrication, characterization and data analysis. R.H.S. conducted PXRD, SCXRD measurements and data analysis. Y.Z. designed the setup and helped with device fabrication. M.B. performed DFT calculations. M.W.T. conducted PDF analysis. D.P. helped with SCXRD refinement. F.N. synthesized and purified compound $\mathbf{1}$ and 1-Br 12 . B.Z., R.H.S., and C.N. wrote the manuscript. Y.Z., M.B., M.W.T., D.P., S.J.L., F.N and M.L.S. contributed to revision of the manuscript. All authors discussed the results and reviewed the manuscript.

\section{Additional information}

Supplementary Information accompanies this paper at https://doi.org/10.1038/s41467018-04246-0.

Competing interests: The authors declare no competing interests.

Reprints and permission information is available online at http://npg.nature.com/ reprintsandpermissions/

Publisher's note: Springer Nature remains neutral with regard to jurisdictional claims in published maps and institutional affiliations.

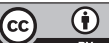

Open Access This article is licensed under a Creative Commons Attribution 4.0 International License, which permits use, sharing, adaptation, distribution and reproduction in any medium or format, as long as you give appropriate credit to the original author(s) and the source, provide a link to the Creative Commons license, and indicate if changes were made. The images or other third party material in this article are included in the article's Creative Commons license, unless indicated otherwise in a credit line to the material. If material is not included in the article's Creative Commons license and your intended use is not permitted by statutory regulation or exceeds the permitted use, you will need to obtain permission directly from the copyright holder. To view a copy of this license, visit http://creativecommons.org/ licenses/by/4.0/.

(C) The Author(s) 2018 